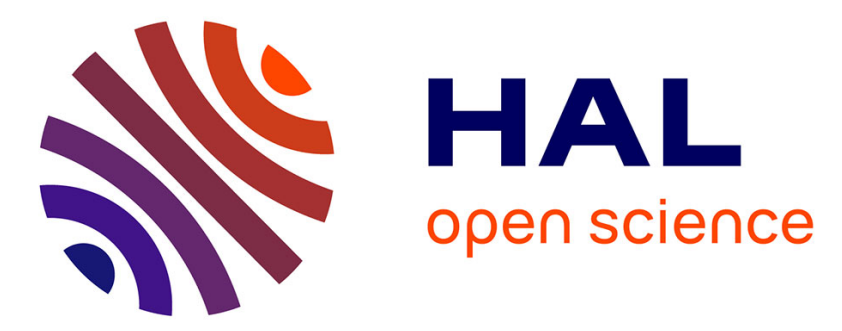

\title{
EM-ESPRIT ALGORITHM FOR DIRECTION FINDING WITH NONUNIFORM ARRAYS
}

\author{
Carine El Kassis, José Picheral, Chafik Mokbel
}

\section{To cite this version:}

Carine El Kassis, José Picheral, Chafik Mokbel. EM-ESPRIT ALGORITHM FOR DIRECTION FINDING WITH NONUNIFORM ARRAYS. Statistical Signal Processing Workshop, Aug 2007, Madison, United States. pp.453-457, 10.1109/SSP.2007.4301299 . hal-00258484

\section{HAL Id: hal-00258484}

https://hal-centralesupelec.archives-ouvertes.fr/hal-00258484

Submitted on 22 Feb 2008

HAL is a multi-disciplinary open access archive for the deposit and dissemination of scientific research documents, whether they are published or not. The documents may come from teaching and research institutions in France or abroad, or from public or private research centers.
L'archive ouverte pluridisciplinaire HAL, est destinée au dépôt et à la diffusion de documents scientifiques de niveau recherche, publiés ou non, émanant des établissements d'enseignement et de recherche français ou étrangers, des laboratoires publics ou privés. 


\section{EM-ESPRIT ALGORITHM FOR DIRECTION FINDING WITH NONUNIFORM ARRAYS}

\author{
Carine El Kassis, José Picheral
}

\author{
Dept. Signal Processing and Electronic Systems, \\ Supélec, Gif-sur-Yvette, France \\ E-mail : firstname.lastname@supelec.fr
}

\author{
Chafic Mokbel
}

University of Balamand, Lebanon

E-mail : chafic.mokbel@balamand.edu.lb

\begin{abstract}
This paper deals with the problem of the Direction Of Arrival (DOA) estimation with nonuniform linear arrays. The proposed method is a combination of the Expectation Maximization (EM) and the ESPRIT methods. The EM algorithm interpolates the nonuniform array to an equivalent uniform array, and then, the application of ESPRIT is possible, in order to estimate the DOA. One of this method novelties lies in its capacity of dealing with any nonuniform array geometry. This technique manifests significant performance and computational advantages over previous algorithms such as MUSIC, specially in the preasymptotic domain, and the comparison with the theoretical Cramer-Rao Bounds (CRB) shows its efficiency.
\end{abstract}

Index Terms - Antenna arrays, nonuniformly spaced arrays, direction of arrival estimation, EM algorithm, ESPRIT.

\section{INTRODUCTION}

The problem of estimating the directions of multiple sources using Nonuniform Linear antenna Arrays (NLA) has attracted considerable attention for both theoretical and practical reasons. Sometimes, using a nonuniform array yields better performance than using a uniform one [1], or the sensors cannot be uniformly spaced due to spatial constraints. Except for spectral MUSIC, high resolution methods cannot be directly applied on NLA because they exploit the uniform geometry of the array. Several approaches have been proposed, among them EM-IQML [1], the interpolated virtual array methods of Friedlander [2], the methods of Higher Order Statistics (HOS) [3] and the positive definite Toeplitz completion [4]. The originality of the proposed method in this paper is the combination of the EM algorithm, to interpolate the data on a Virtual Uniform Linear Array (VULA), with the ESPRIT method used for DOA estimation. In fact, the NLA output is treated as incomplete data which makes the EM algorithm directly applicable, in an iterative way. The interpolation is based on the signal model. Hence, the error due to interpolation is reduced iteratively when the accuracy of parameter estimation improves. This error always exists in [2]. ESPRIT is a simple and accurate method for DOA estimation that achieves a significant reduction in computational complexity in comparison with IQML and HOS. Also, ESPRIT presents better performance in the preasymptotic domain than IQML. Another advantage of the proposed method is its ability to deal with any type of nonuniform arrays. It can be applied to sublattice arrays, which are not necessarily minimum redundancy arrays, and to nonregular linear arrays, where the intersensor separation is chosen in an arbitrary way.

This paper is organized as follows. The signal model is presented in Section 2. In Section 3, the EM-ESPRIT algorithm is described for both types of nonuniform arrays. Simulation results are presented in Section 4 and the main conclusions drawn from them are summarized in Section 5.

\section{SIGNAL MODEL}

Consider $N$ far-field narrowband sources incident on an $M$ elements linear array, from directions $\boldsymbol{\theta}=\left[\theta_{1}, \ldots, \theta_{N}\right]^{\top}$. The sensors, assumed to be omnidirectional, are situated at positions $d_{m}(m=1, \ldots, M)$. Two kinds of NLA are considered: $i$ ) the sublattice array, which can be considered as a ULA where some elements are omitted, i.e. $d_{m}=k_{m} \Delta$ where $\Delta$ is the ULA intersensor separation and $k_{m}$ is an integer, and $\mathrm{ii}$ ) the nonregular linear array, where the intermediate distances between sensors are chosen in an arbitrary way. Without loss of generality, $\Delta$ is taken as the half-wavelength.

Grouping the signals received by the $M$ array elements in the $M \times 1$ vector $\mathbf{y}(t)$, the sensor outputs can be written as:

$$
\mathbf{y}(t)=\mathbf{A}(\boldsymbol{\theta}) \mathbf{s}(t)+\boldsymbol{\nu}(t)
$$

where $\mathbf{A}(\boldsymbol{\theta})=\left[\mathbf{a}\left(\theta_{1}\right), \ldots, \mathbf{a}\left(\theta_{N}\right)\right]$ is the $M \times N$ steering matrix and $\mathbf{a}\left(\theta_{n}\right)$ is the steering vector of the $n$-th source:

$$
\mathbf{a}\left(\theta_{n}\right)=\left[e^{-j 2 \pi \frac{d_{1} \sin \theta_{n}}{\lambda}}, \ldots, e^{-j 2 \pi \frac{d_{M} \sin \theta_{n}}{\lambda}}\right]^{\top} .
$$

The $N \times 1$ vector $\mathbf{s}(t)$ contains the complex amplitude of the incident signals. The $M \times 1$ vector $\boldsymbol{\nu}(t)$ represents a Gaussian additive noise, which is zero mean and such as $E\left\{\boldsymbol{\nu}(t) \boldsymbol{\nu}(t)^{H}\right\}=\sigma^{2} \mathbf{I}$.

The received signal is sampled, and the received data $\mathbf{Y}=\left[\mathbf{y}\left(t_{1}\right), \ldots, \mathbf{y}\left(t_{L}\right)\right]$ can be expressed as:

$$
\mathbf{Y}=\mathbf{A}(\boldsymbol{\theta}) \mathbf{S}+\mathbf{V}
$$


where $L$ is the number of samples, $\mathbf{S}=\left[\mathbf{s}\left(t_{1}\right), \ldots, \mathbf{s}\left(t_{L}\right)\right]$ and $\mathbf{V}=\left[\boldsymbol{\nu}\left(t_{1}\right), \ldots, \boldsymbol{\nu}\left(t_{L}\right)\right]$.

\section{DOA ESTIMATION}

Since the nonuniform array outputs can be treated as incomplete data, the EM algorithm approach can be applied to our case. The main idea consists of interpolating the sparse array in a way that a uniform array is realized and then estimating the DOA through ESPRIT applied to the new VULA. We first start by studying the case of the sublattice array (case $i)$ ).

\subsection{Data construction}

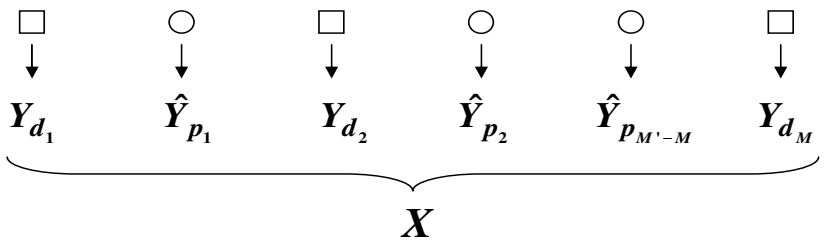

Fig. 1. Example of NLA and its equivalent VULA, with $\mathbf{d}=[1,3,6] \frac{\lambda}{2}, \mathbf{p}=[2,4,5] \frac{\lambda}{2}$ and $\ell=[1,0,1,0,0,1]$. $\square$ : existing sensors $(\mathbf{Y}), \mathrm{O}$ : omitted sensors $\left(\hat{\mathbf{Y}}_{\mathbf{p}}\right)$.

Consider the VULA of $M^{\prime}$ sensors $\left(M^{\prime} \geq M\right)$ formed by the sensors of the NLA and by the omitted virtual sensors as represented in Fig. 1 . Let $\mathbf{X}=\left[x\left(t_{1}\right), \ldots, x\left(t_{L}\right)\right]$ be defined as the unavailable data output of the VULA. The NLA output $\mathbf{Y}$ can be considered as an incomplete data observation of $\mathbf{X}$ and the EM is directly applicable. The linear transformation mapping $\mathbf{X}$ to $\mathbf{Y}$ is given by:

$$
\mathbf{Y}=\mathbf{G}^{\top} \mathbf{X}
$$

By construction, the noise free parametric model of $\mathrm{X}$ is given by:

$$
\boldsymbol{\mu}(\boldsymbol{\theta})=\left(\mathbf{G A}(\boldsymbol{\theta})+\overline{\mathbf{G}} \mathbf{A}_{\mathbf{p}}(\boldsymbol{\theta})\right) \mathbf{S} .
$$

The $M^{\prime} \times M$ matrix $\mathbf{G}$ is constructed by eliminating the zero columns from $\operatorname{diag}(\ell)$, where the $M^{\prime} \times 1$ vector $\ell$ describes the binary transformation between $\mathbf{X}$ and $\mathbf{Y}$ : the $m$-th component of $\ell$ is 1 if the $m$-th sensor of the VULA is part of the NLA, and 0 otherwise. The $M^{\prime} \times\left(M^{\prime}-M\right)$ matrix $\overline{\mathbf{G}}$ describes the relation between $\mathbf{X}$ and the missing data, it is constructed similarly to $\mathbf{G}$. $\mathbf{A}_{\mathbf{p}}(\boldsymbol{\theta})$ is the steering matrix of the omitted sensors. Let the vector $\mathbf{p}=\left[p_{1}, \ldots, p_{M^{\prime}-M}\right]$ of length $\left(M^{\prime}-M\right)$ represent the positions of the omitted sensors. Then, $\mathbf{A}_{\mathbf{p}}(\boldsymbol{\theta})$ can be written as:

$$
\begin{aligned}
\mathbf{A}_{\mathbf{p}}(\boldsymbol{\theta}) & =\left[\mathbf{a}_{\mathbf{p}}\left(\theta_{1}\right), \ldots, \mathbf{a}_{\mathbf{p}}\left(\theta_{N}\right)\right], \\
\mathbf{a}_{\mathbf{p}}\left(\theta_{n}\right) & =\left[e^{-j 2 \pi \frac{p_{1} \sin \theta_{n}}{\lambda}}, \ldots, e^{-j 2 \pi \frac{p_{M^{\prime}-M} \sin \theta_{n}}{\lambda}}\right]^{\top} .
\end{aligned}
$$

Notice that the model $\boldsymbol{\mu}(\boldsymbol{\theta})$ can be rewritten as

$$
\boldsymbol{\mu}(\boldsymbol{\theta})=\mathbf{A}_{V U L A}(\boldsymbol{\theta}) \mathbf{S},
$$

where $\mathbf{A}_{V U L A}(\boldsymbol{\theta})$ is the array response of a uniform linear array, in this case, the VULA.

\subsection{The EM general approach}

Basically, EM is an iterative approach of the maximum likelihood estimator (MLE). Each iteration is composed of two steps: the Expectation step (E-step) and the Maximization step (M-step). In the E-step, the conditional likelihood of the complete data $\hat{\mathbf{X}}^{(k)}$, given the incomplete data $\mathbf{Y}$ and the previous estimate of the parameters $\hat{\boldsymbol{\theta}}^{(k-1)}$, is estimated. In the $\mathrm{M}$-step, the parameters $\hat{\boldsymbol{\theta}}^{(k)}$ are estimated by maximizing the conditional likelihood criterion. It can be shown that $\hat{\boldsymbol{\theta}}^{(k)}$ converges to the MLE (see [5]).

A justification of the EM algorithm is as follows. Using Bayes' rule, and taking the logarithm of the probability densities,

$$
\ln f_{\mathbf{y}}(\mathbf{Y} \mid \boldsymbol{\theta})=\ln f_{\mathbf{x}}(\mathbf{X} \mid \boldsymbol{\theta})-\ln f_{\mathbf{x} \mid \mathbf{Y}}(\mathbf{X} \mid \mathbf{Y}, \boldsymbol{\theta}) .
$$

Taking the conditional expectation of (8) given $\mathbf{Y}$ at a parameter value $\boldsymbol{\theta}^{\prime}$ yields

$$
L(\boldsymbol{\theta}) \triangleq \ln f_{\mathbf{y}}(\mathbf{Y} \mid \boldsymbol{\theta})=U\left(\boldsymbol{\theta} \mid \boldsymbol{\theta}^{\prime}\right)-V\left(\boldsymbol{\theta} \mid \boldsymbol{\theta}^{\prime}\right),
$$

where

$$
\begin{aligned}
& U\left(\boldsymbol{\theta} \mid \boldsymbol{\theta}^{\prime}\right)=E\left\{\ln \left\{f_{\mathbf{x}}(\mathbf{X} \mid \boldsymbol{\theta})\right\} \mid \mathbf{Y}, \boldsymbol{\theta}^{\prime}\right\} \\
& V\left(\boldsymbol{\theta} \mid \boldsymbol{\theta}^{\prime}\right)=E\left\{\ln \left\{f_{\mathbf{x} \mid \mathbf{Y}}(\mathbf{X} \mid \mathbf{Y}, \boldsymbol{\theta})\right\} \mid \mathbf{Y}, \boldsymbol{\theta}^{\prime}\right\}
\end{aligned}
$$

$L(\boldsymbol{\theta})$, the log-likelihood of the observed data, is the function to be maximized. Knowing that $V\left(\boldsymbol{\theta} \mid \boldsymbol{\theta}^{\prime}\right) \leq V\left(\boldsymbol{\theta}^{\prime} \mid \boldsymbol{\theta}^{\prime}\right)$ (Jensen's inequality), if $U\left(\boldsymbol{\theta} \mid \boldsymbol{\theta}^{\prime}\right)>U\left(\boldsymbol{\theta}^{\prime} \mid \boldsymbol{\theta}^{\prime}\right)$, then $L(\boldsymbol{\theta})>L\left(\boldsymbol{\theta}^{\prime}\right)$. Thus, the maximization of $U\left(\boldsymbol{\theta} \mid \boldsymbol{\theta}^{\prime}\right)$ improves the ML criterion. Exploiting this property, the EM algorithm can be described as follows.

It starts with an initial guess $\hat{\boldsymbol{\theta}}^{(0)}$, and maximizes $L(\boldsymbol{\theta})$ by iterating the $\mathrm{E}$ and $\mathrm{M}$-steps, i.e. at iteration $k$ :

E-step: Compute $U\left(\boldsymbol{\theta} \mid \hat{\boldsymbol{\theta}}^{(k-1)}\right)$.

M-step: Estimate $\hat{\boldsymbol{\theta}}^{(k)}$ as $\hat{\boldsymbol{\theta}}^{(k)}=\arg \max _{\boldsymbol{\theta}} U\left(\boldsymbol{\theta} \mid \hat{\boldsymbol{\theta}}^{(k-1)}\right)$.

\subsection{EM-ESPRIT}

Now, we apply EM to our case. We show that the E-step is equivalent to estimating the outputs of the omitted elements, using an interpolation based on the signal model. In the Mstep, the DOA are estimated using ESPRIT. While in the Estep the proposed approach follows the classical EM estimation, the ESPRIT algorithm in the M-step is a sub-optimal method in comparison to ML. These steps are explained in more details in the following.

E-step: As shown in [1], the maximization of $U\left(\boldsymbol{\theta} \mid \hat{\boldsymbol{\theta}}^{(k-1)}\right)$ reduces to the minimization of $\left\|\hat{\mathbf{X}}^{(k)}-\boldsymbol{\mu}(\boldsymbol{\theta})\right\|^{2}$, where

$$
\hat{\mathbf{X}}^{(k)}=E\left\{\mathbf{X} \mid \mathbf{Y} ; \hat{\boldsymbol{\theta}}^{(k-1)}\right\} .
$$


Thus, we only need to find $\hat{\mathbf{X}}^{(k)}$ instead of computing $U\left(\boldsymbol{\theta} \mid \hat{\boldsymbol{\theta}}^{(k-1)}\right)$. Using (5) and the results of [6], $\hat{\mathbf{X}}^{(k)}$ is given by:

$$
\begin{aligned}
\hat{\mathbf{X}}^{(k)} & =\mathbf{G A}\left(\hat{\boldsymbol{\theta}}^{(k-1)}\right) \hat{\mathbf{S}}^{(k-1)}+\overline{\mathbf{G}} \mathbf{A}_{\mathbf{p}}\left(\hat{\boldsymbol{\theta}}^{(k-1)}\right) \hat{\mathbf{S}}^{(k-1)} \\
& +\mathbf{G}\left[\mathbf{G}^{H} \mathbf{G}\right]^{-1}\left(\mathbf{Y}-\mathbf{A}\left(\hat{\boldsymbol{\theta}}^{(k-1)}\right) \hat{\mathbf{S}}^{(k-1)}\right) .
\end{aligned}
$$

Since $\left[\mathbf{G}^{H} \mathbf{G}\right]^{-1}=I$, (11) becomes:

$$
\hat{\mathbf{X}}^{(k)}=\mathbf{G} \mathbf{Y}+\overline{\mathbf{G}} \mathbf{A}_{\mathbf{p}}\left(\hat{\boldsymbol{\theta}}^{(k-1)}\right) \hat{\mathbf{S}}^{(k-1)},
$$

where $\hat{\mathbf{S}}^{(k)}$ is estimated by $\hat{\mathbf{S}}^{(k)}=\mathbf{A}^{\dagger}\left(\hat{\boldsymbol{\theta}}^{(k)}\right) \mathbf{Y}$, and the symbol ()$^{\dagger}$ represents the pseudo-inverse.

Thus, the complete data $\hat{\mathbf{X}}^{(k)}$ is equal to the measured data $\mathbf{Y}$ for the rows corresponding to the existing sensors, and for the rows of the missing data, the data are interpolated using the parametric model $\boldsymbol{\mu}(\boldsymbol{\theta})$. Let $\hat{\mathbf{Y}}_{\mathbf{p}}^{(k)}=\mathbf{A}_{\mathbf{p}}\left(\hat{\boldsymbol{\theta}}^{(k-1)}\right) \hat{\mathbf{S}}^{(k-1)}$ be the interpolated missing data.

The noise contribution in $\hat{\mathbf{Y}}_{\mathbf{p}}^{(k)}$ is omitted, because its expectation is null since the noise is supposed to be zero mean.

M-step: Since the new constructed array is uniform, any conventional method for DOA estimation in the case of uniform arrays can be used. This is justified by (7). Thus, DOA estimation $\hat{\boldsymbol{\theta}}^{(k)}$ can be done by applying the conventional ESPRIT method. Therefore, we need to calculate the VULA data correlation matrix, $\hat{\mathbf{R}}^{(k)}$. It is estimated using $\hat{\mathbf{X}}^{(k)}$ and the noise contribution on the interpolated data modeled by $\overline{\mathbf{G}} \overline{\mathbf{G}}^{H} \hat{\sigma}^{2}$, assuming that the noise contribution in $\hat{\mathbf{Y}}_{\mathbf{p}}^{(k)}$ has the same statistical properties as $\boldsymbol{\nu}(t)$ :

$$
\hat{\mathbf{R}}^{(k)}=\frac{1}{L} \hat{\mathbf{X}}^{(k)} \hat{\mathbf{X}}^{(k) H}+\overline{\mathbf{G}} \overline{\mathbf{G}}^{H} \hat{\sigma}^{2},
$$

where $\hat{\sigma}^{2}$ is a consistent estimate of $\sigma^{2}$ obtained by averaging the smallest eigenvalues of the $\mathbf{Y}$ covariance matrix.

ESPRIT can be used to exploit the translational invariance structure of the covariance data of the VULA, $\hat{\mathbf{R}}^{(k)}$.

To summarize, the proposed algorithm can be presented as follows:

1) Initialization: $k=0$, choose $\hat{\boldsymbol{\theta}}^{(0)}$ and estimate the noise variance $\hat{\sigma}^{2}$.

2) Estimate the complete data $\hat{\mathbf{X}}^{(k)}$ using (12) and estimate the covariance matrix $\hat{\mathbf{R}}^{(k)}$ using (13).

3) Use ESPRIT to estimate $\hat{\boldsymbol{\theta}}^{(k)}$.

4) Check convergence of $\boldsymbol{\theta}$. If not, $k=k+1$, go to step 2.

\subsection{Extension to the nonregular case}

One of the main advantages of the proposed method is its capacity of being extended to the case of nonregular arrays (case $i i)$ ). In this case, the construction of the VULA is different, leading to a change in the construction of the complete data $\mathbf{X}$. With respect to the previous case, the VULA is constructed by only using the data outputs of the first and last sensors of the NLA, and interpolating the other $\left(M^{\prime}-2\right)$ data. The virtual sensors are uniformly spaced. The array aperture remains unchanged and the number of sensors in the VULA, $M^{\prime}$, can be chosen arbitrarily $\left(M^{\prime}>N\right)$. In this case, $\mathbf{G}$ is an $M^{\prime} \times 2$ matrix and $\mathbf{p}$ is an $\left(M^{\prime}-2\right)$ vector. Once the construction of $\mathbf{X}$ completed, the algorithm mentioned above can be applied for the DOA estimation.

Remark: The construction of $\mathbf{X}$ proposed for the case ii) can also be used for the case $i$ ).

\section{SIMULATION RESULTS}

Some simulations have been conducted, exploring different aspects of EM-ESPRIT and making comparisons with other techniques. The results are based on 500 trials in each case. The maximum number of iterations is 30 , but in fact, in all the experiments, not more than 10 iterations are needed for the convergence, as it will be seen later. 500 snapshots are used. In almost all the experiments, the initialization of the angles is done using a simple beamforming.

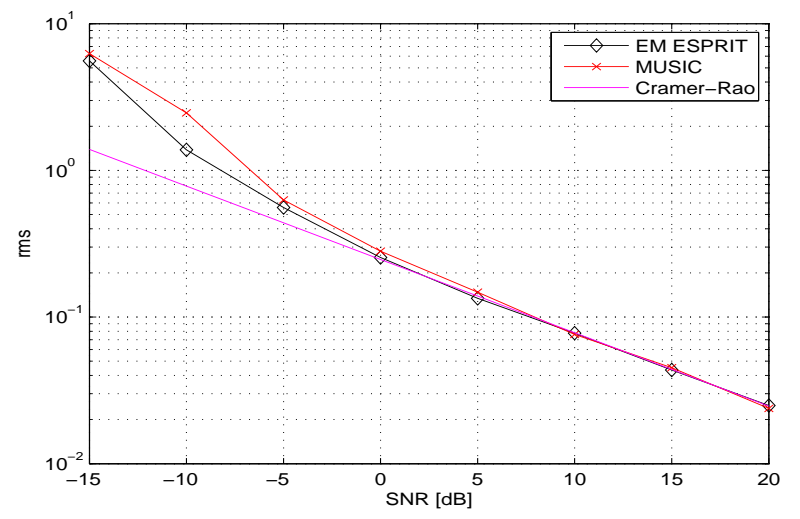

Fig. 2. RMS error for the source at $-5^{\circ}$ for the array $\mathbf{d}=[1,3,6] \frac{\lambda}{2}, N=2$ sources.

In the first set of experiments, different array geometries are used to test the capability of the new method to estimate the DOA. Consider the subarray described by $\mathbf{d}=[1,3,6] \frac{\lambda}{2}$, where the set of intersensor separations has missing lags, the nonregular array given by $\mathbf{d}=[1,2.32,4.03,6] \frac{\lambda}{2}$ and the $\mathbf{d}=[1,2,5,8,14,16,19,24] \frac{\lambda}{2}$ array. The narrowband signals are generated by two and three sources of equal power located respectively at $\left[-5^{\circ}, 10^{\circ}\right]$ and $\left[-5^{\circ}, 10^{\circ}, 45^{\circ}\right]$. The Root Mean Square (RMS) error for the first source is plotted in function of the Signal-Noise Ratio (SNR). First, consider the two sources case. Fig. 2 shows the results for the first array, while the nonregular array results are shown in Fig. 3. The accuracy of the proposed method is compared to spectral MUSIC and to CRB. The CRB was calculated using the 
equations of [7], extended to the case of nonuniform arrays.

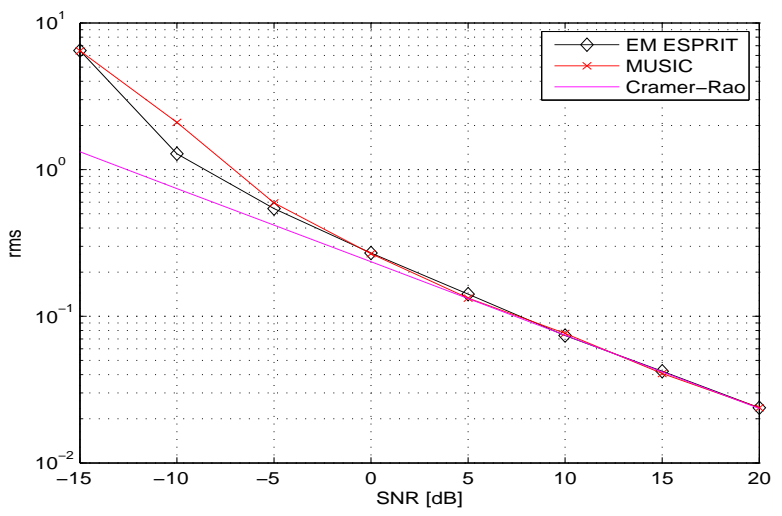

Fig. 3. RMS error for the source at $-5^{\circ}$ for the array $\mathbf{d}=[1,2.32,4.03,6] \frac{\lambda}{2}, N=2$ sources.

We notice that EM-ESPRIT is a consistent method and it shows better performance in comparison to MUSIC, specially in the preasymptotic domain, with lower complexity.

Now, the results of the last angle $\left(45^{\circ}\right)$ are plotted in Fig. 4 , using the array $\mathbf{d}=[1,2,5,8,14,16,19,24] \frac{\lambda}{2}$, in the case of three sources. We notice that in this situation also, the EMESPRIT method is consistent, and performs almost as well as MUSIC for the preasymptotic domain. Similar results are obtained for the other sources. Thus, we can conclude that EM-ESPRIT is suitable for any type of array geometry.

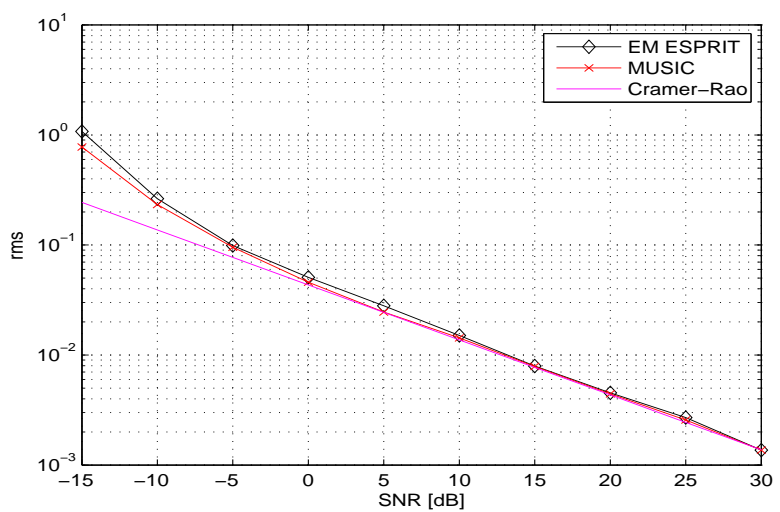

Fig. 4. RMS error for the source at $45^{\circ}$ for the array $\mathbf{d}=[1,2,5,8,14,16,19,24] \frac{\lambda}{2}, N=3$ sources.

For the second experiment, we use the $\mathbf{d}=[1,3,6] \frac{\lambda}{2}$ geometry, since similar results are obtained for other geometries. The resolution of the method is tested. We consider the case of two sources, where the fixed angle is $0^{\circ}$ and the second angle is separated by $\Delta \theta . \Delta \theta$ varies from $2^{\circ}$ to $20^{\circ}$. The SNR is fixed to $10 \mathrm{~dB}$. Only the second angle is presented in Fig. 5.

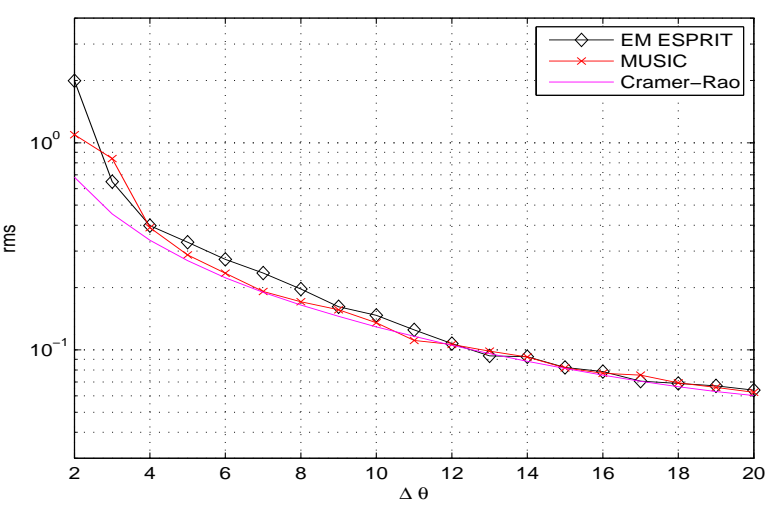

Fig. 5. Resolution for the second source of the array $\mathbf{d}=[1,3,6] \frac{\lambda}{2}$, at $\mathrm{SNR}=10 \mathrm{~dB}, N=2$ sources.

EM-ESPRIT presents good resolution, even for close angle values. This result is expected, since ESPRIT is a highresolution method.

In the third type of experiments, we test different types of initialization, to check its influence on the performance. The array used is $\mathbf{d}=[1,2,4,6] \frac{\lambda}{2}$. We consider the case of two sources located at $\left[-5^{\circ}, 10^{\circ}\right]$. The results for the source at $-5^{\circ}$ are shown at Fig. 6, where the RMS error is plotted as a function of the SNR. Three types of initialization are compared: the beamforming, the real values $\left[-5^{\circ}, 10^{\circ}\right]$ and arbitrary values taken at $\left[0^{\circ}, 40^{\circ}\right]$.

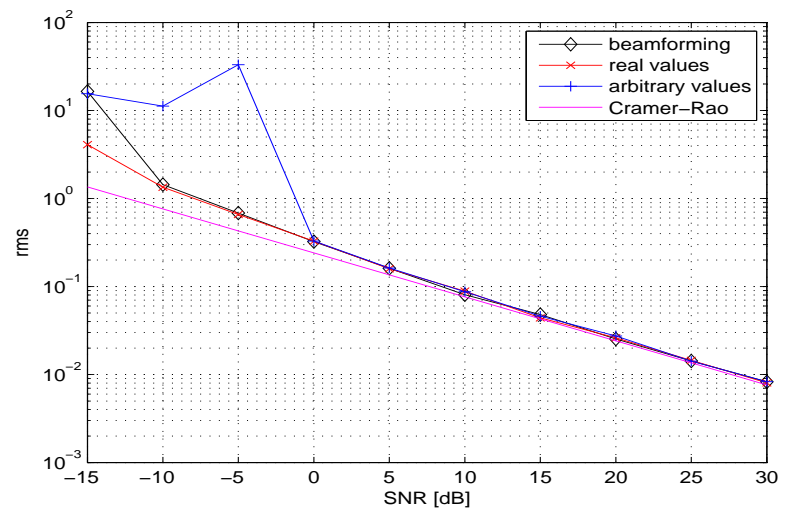

Fig. 6. RMS error for the source at $-5^{\circ}$ for the array $\mathbf{d}=[1,2,4,6] \frac{\lambda}{2}$, using different kinds of initialization, $N=$ 2 sources.

This result shows that EM-ESPRIT is not highly sensitive to the accuracy of the initialization. At low SNR, the performances are almost the same when using beamforming or the exact values, and they decrease when using arbitrary values far from the exact ones. At high SNR, performances become independent of the initialization. Similar results are obtained for the source at $10^{\circ}$, and for other geometries. 


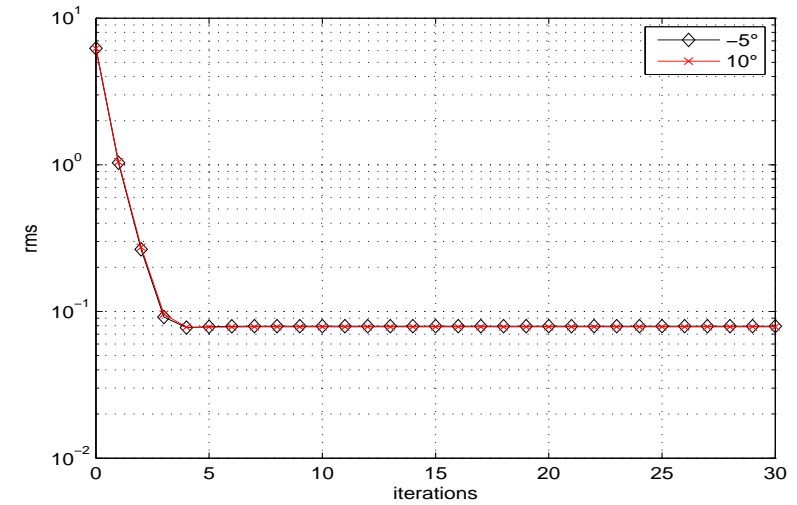

Fig. 7. RMS error for the sources at $-5^{\circ}$ and $-10^{\circ}$ for the array $\mathbf{d}=[1,2.32,4.03,6] \frac{\lambda}{2}$, in function of the number of iterations, $N=2$ sources.

Next, we investigate the convergence of EM-ESPRIT. We use the array defined by $\mathbf{d}=[1,2.32,4.03,6] \frac{\lambda}{2}$, at $\mathrm{SNR}=$ $10 \mathrm{~dB}$, and we consider two cases: two sources generating at $\left[-5^{\circ}, 10^{\circ}\right]$, and three sources located at $\left[-5^{\circ}, 10^{\circ}, 45^{\circ}\right]$. The RMS error for the two angles of the first case is drawn in function of the number of iterations in Fig. 7, while the RMS error of the three sources of the second case are shown in Fig. 8. Iteration 0 means the initialization value obtained with the beamforming method.

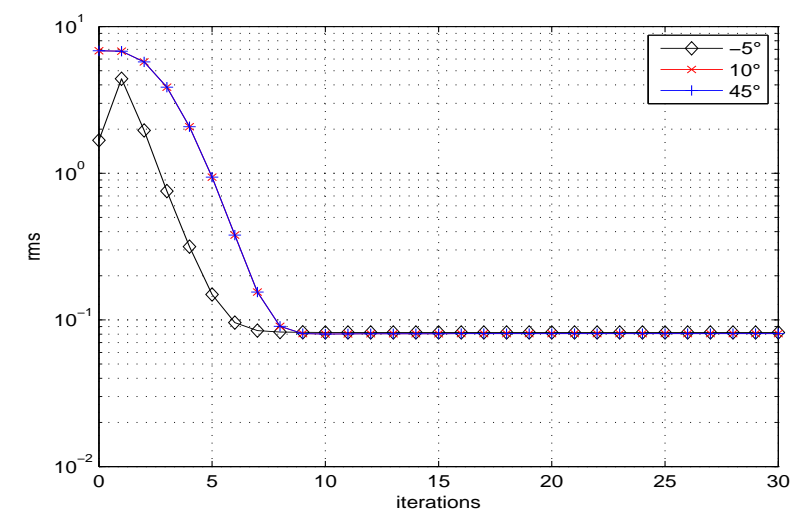

Fig. 8. RMS error for the sources at $-5^{\circ}, 10^{\circ}$ and $45^{\circ}$ for the array $\mathbf{d}=[1,2.32,4.03,6] \frac{\lambda}{2}$, in function of the number of iterations, $N=3$ sources.

It can be seen that EM-ESPRIT converges in few iterations, even though in the case of three sources the convergence is slower. But in general, not more than 10 iterations are needed, which means that EM-ESPRIT converges fast, and the computation time is reduced. Similar results are obtained at other SNR values.

\section{CONCLUSION}

In this paper, an EM-ESPRIT method for the estimation of narrowband sources observed by nonuniform arrays is proposed. The algorithm is efficient and consistent. It even outperforms other methods in the preasymptotic domain and for lower computational cost. This method has the advantage of dealing with all types of nonuniform arrays, where other methods fail to treat the nonregular case, or the sublattice arrays that are not minimum redundancy ones.

\section{REFERENCES}

[1] A. J. Weiss, A. S. Willsky, and B. C. Levy, "Nonuniform array processing via the polynomial approach," IEEE Trans. Aerosp. Electron. Syst., vol. 25, no. 1, pp. 48-55, January 1989.

[2] B. Friedlander, "The root-MUSIC algorithm for direction finding with interpolated arrays," Signal Process., vol. 30, no. 1, pp. 15-29, January 1993.

[3] Y. H. Chen and Y. S. Lin, "Direction finding with nonuniform arrays via higher-order statistics," IEEE Antennas and Propagation Society International Symposium, vol. 3, pp. 1576-1579, 1994.

[4] Y. I. Abramovich, D. A. Gray, A. Y. Gorokhov, and N. K. Spencer, "Positive-definite Toeplitz completion in DOA estimation for nonuniform linear antenna arrays-part I: fully augmentable arrays," IEEE Trans. Signal Processing, vol. 46, no. 9, pp. 2458-2471, September 1998.

[5] A. P. Dempster, N. M. Laird, and D. B. Rubin, "Maximum likelihood from incomplete data via the EM algorithm," Royal Statistical Society, series B, vol. 39, pp. 1-38, 1977.

[6] M. Feder and E. Weinstein, "Parameter estimation of superimposed signals using the EM algorithm," IEEE Trans. Acoust., Speech, Signal Processing, vol. 36, no. 4, pp. 477-489, April 1988.

[7] P. Stoica and A. Nehorai, "MUSIC, maximum likelihood, and cramer-rao bound," IEEE Trans. Acoust., Speech, Signal Processing, vol. 37, no. 5, pp. 720-741, May 1989. 